\title{
ON THE EXISTENCE OF PERIODIC SOLUTION OF SOME QUASILINEAR DIFFERENTIAL EQUATIONS WITH IMPULSES
}

\author{
LE LUONG TAI \\ Thai Nguyen University
}

\section{Introduction}

As for ordinary differential equations, one of the problems that especially attract the attention of many mathematicians is the problem on the existence of periodic solutions of the differential equation systems with impulses. In this paper, we study the periodic solutions of the equation system under of the form:

$$
\begin{aligned}
& \dot{x}=A(x, t) x+F(t, x), \quad t \neq \tau_{i}, \\
& \left.\Delta x\right|_{t=\tau_{i}}=B_{i}(x) x+c_{i}(x), \\
& B_{i+p}(x)=B_{i}(x), \quad c_{i+p}=c_{i}(x), \quad p \text {-integers, } \quad i=1,2, \ldots
\end{aligned}
$$

where $A(t, x), B_{i}(x)$ are $n \times n$-matrices, $F(t, x), c_{i}(x)$ are $n$-vector, the elements of which are continuous with respect to its variables (piecewise continuous with the first kind discontinuities in $t$ at $t=\tau_{i}$ ) and $T$-periodic with respect to $t$.

In the case of ordinary differential equations, this problem was discussed by A. G. Kartsatos [1], S. Saito and M. Yamamoto [2]. Here this result will be extended to the case of ordinary differential equations with impulses.

Together with the system (1.1) and (1.2), we consider the homogeneous linear differential equation system with impulses

$$
\begin{aligned}
& \dot{x}=A_{0}(t) x, \quad t \neq \tau_{i}, \\
& \left.\Delta x\right|_{t=\tau_{i}}=B_{i}^{0} x, \quad B_{i+p}^{0}=B_{i}^{0}, \quad i=1,2, \ldots,
\end{aligned}
$$

where $A_{0}(t)$ is a continuous $T$-periodic in $t$ matrix; $B_{i}^{0}$ are the constant matrices.

The following symbols are introduced. We denote by $\|\cdot\|$ a norm of a vector or matrix, and by the symbol $\|\cdot\|_{0}$ a supremum norm of vector or matrix function respectively. For example, for the vector function $\varphi(t)$ and the matrix function $A(t)$ we have

$$
\|\varphi(t)\|_{0}=\sup _{t \in[0, T]}\|\varphi(t)\|, \quad\|A(t)\|_{0}=\sup _{t \in[0, T]}\|A(t)\| .
$$

Two of following problems will be solved in this work: 
Problem 1. Suppose that the system (1.4) and (1.5) does not have nontrivial $T$-periodic solutions i.e. $H_{0}=E-X_{0}(T)$ has an inverse matrix $H_{0}^{-1}$. The question here is that in what conditions the system of equations

$$
\begin{aligned}
& \dot{x}=A(t) x, \quad t \neq \tau_{i}, \\
& \left.\Delta x\right|_{t=\tau_{i}}=B_{i} x, \quad B_{i+p}=B_{i}, \quad i=1,2, \ldots
\end{aligned}
$$

also does not have nontrivial T-periodic solutions, i.e. $H=E-X(T)$ has an inverse matrix $H^{-1}$, where $X_{0}(t)$ and $X(t)$ respectively are the matriciants of the system (1.4), (1.5) and of (1.6), (1.7)? Moreover, under what conditions that the T-periodic vector function $f(t)$ and vectors $c_{i}$, should be satisfied the corresponding non homogeneous linear equation system

$$
\begin{aligned}
& \dot{x}=A(t) x+f(t), \quad t \neq \tau_{i}, \\
& \left.\Delta x\right|_{t=\tau_{i}}=B_{i} x+c_{i}, \quad B_{i+p}=B_{i}, \quad c_{i+p}=c_{i}, \quad i=1,2, \ldots
\end{aligned}
$$

has a $T$-periodic solution that belongs to the region $\|x\| \leq h$.

Problem 2. Using the assumptions stated in Problem 1 for the system (1.4), (1.5), let us examine under what conditions the equation system (1.1), (1.2), (1.3) has a T-periodic solution.

Before giving out statements of the main results of the work, some fundamental knowledges on periodic linear differential equation system with impulses of the form (1.6), (1.7) are presented and some symbols to be used afterwards are introduced as follows

1) The equation system (1.6) and (1.7) has a fundamental matrix of solutions $X(t)$ :

$$
X(t)=U\left(t, \tau_{k+1}\right)\left(E+B_{k+1}\right) U\left(\tau_{k+1}, \tau_{k}\right)\left(E+B_{k}\right) \cdots\left(E+B_{1}\right) U\left(\tau_{1}, t_{0}\right) X\left(t_{0}\right),
$$

where $0 \leq t_{0} \leq \tau_{1}<\tau_{k}<t \leq \tau_{k+1}$; and $U(t, s)$ is a solution of Cauchy problem

$$
\frac{d U}{d t}=A(t) U, \quad U(s, s)=E .
$$

For the differential equation with impulses the matrix $X(t)$, that $X\left(t_{0}\right)=E$ is also called a matriciant of the system.

2) When investigating the problem on the existence of periodic solutions, in the general case let use the operator

$$
V(x(.))=x(0)-x(T),
$$

and in the case of the linear differential equations, we apply the operator

$$
H=I-X(T) .
$$

For the solution $x(t), x\left(t_{0}\right)=x_{0}$ of the linear differential equations, the operator $V(x(t))$ becomes

$$
V(x(t))=H x_{0}
$$


Consequently, if $\operatorname{det} H \neq 0$ we obtain also

$$
x_{0}=H^{-1} V(x(t)) .
$$

3) The following symbols will be used

$$
\begin{aligned}
& K=\exp \left(\int_{0}^{T}\left\|A_{0}(s)\right\| d s\right),\left\|B_{i}^{0}\right\|=\beta_{i}, \prod_{i=1}^{p}\left(1+\beta_{i}\right)=L, \prod_{i=1}^{p}\left(1+\beta_{i}+\delta_{1}\right)=L_{1}, \\
& \left\|\left(E+B_{i}^{0}\right)^{-1}\right\|=\ell_{i}, \quad \prod_{i=1}^{p}\left(1+\beta_{i} \ell_{i}\right)=M, \quad \prod_{i=1}^{p}\left(1+\frac{\ell_{i}\left(\beta_{i}+\delta_{1}\right)}{1-\ell_{i} \delta_{1}}\right)=M_{1} .
\end{aligned}
$$

4) In this paper, the system (1.4) and (1.5) are taken into the system to compare with considered systems. It is assumed that, the system (1.4) and (1.5) does not have $T$-periodic nontrivial solutions. In connection with this system, indexes 0 are added to symbols as subscripts. Thus, we have

$$
\begin{aligned}
X_{0}(t) & =E+\int_{0}^{t} A_{0}(s) X_{0}(s) d s+\sum_{0<\tau_{i}<t} B_{i}^{0} X_{0}\left(\tau_{i}\right) \\
X_{0}^{-1}(t) & =E-\int_{0}^{T} X_{0}^{-1}(s) A_{0}(s) d s-\sum_{0<\tau_{i}<t} X_{0}^{-1}\left(\tau_{i}\right) B_{i}^{0}\left[\left(E+B_{i}^{0}\right)^{-1}\right] .
\end{aligned}
$$

By using extended Gronwall inequality [3], we get

$$
\left\|X_{0}(t)\right\| \leq L K, \quad X_{0}^{-1}(t) \leq M K .
$$

Moreover, if the system (1.4), (1.5) does not have nontrivial $T$-periodic solutions, then $H_{0}=I-X_{0}(T)$ has an inverse matrix $H_{0}^{-1} \cdot[2]$ and, a positive number $\rho, 0<\rho<1$ can be chosen such that

$$
\left\|H_{0}^{-1}\right\| \leq \frac{1}{\rho}
$$

\section{The main results}

Theorem 1. Suppose that $A(t)$ and $f(t)$ correspondently are T-periodic, piecewise continuous with the first kind discontinuities in $t$ at $t=\tau_{i}$ matrix and vector functions, $B_{i}$, $c_{i}$ correspondently are the constant matrix and vector. Moreover, the matrices $A(t), B_{i}$ satisfy the inequalities

$$
\left\|A(t)-A_{0}(t)\right\| \leq \delta, \quad\left\|B_{i}-B_{i}^{0}\right\| \leq \delta_{1},
$$

with $\delta, \delta_{1}$ satisfied the inequality

$$
M L^{2} K^{3}\left(T \delta+p \delta_{1}\right)\left(1+L M K^{2} \delta_{1}\right)^{p} \exp \left(M L K^{2} T \delta\right) \leq \frac{\rho}{2\left\|H_{0}^{-1}\right\|},
$$


where $0<\rho<1$. If the equation system (1.4), (1.5) does not have nontrivial T-periodic solutions, then

i) The matrix $H=E-X(T)$ has an inverse matrix $H^{-1}$ that satisfies the condition

$$
\left\|H^{-1}\right\| \leq \frac{1}{\rho(1-\rho)} .
$$

ii) For every T-periodic, piecewise continuous with the first kind discontinuities at $t=\tau_{i}$ vector function $f(t)$ and $c_{i}, i=1,2, \ldots, p$, that satisfy the condition

$$
N \leq \frac{\rho(1-\rho) h}{\left[2 L_{1} M_{1} K^{2} \exp (T \delta)+\rho(1-\rho)\right] L_{1} K \exp (T \delta)},
$$

where $N=\max \left\{\int_{0}^{T}\|f(s)\| d s, \sum_{i=1}^{p}\left\|c_{i}\right\|\right\}$, the equation system (1.6), (1.7) has a T-periodic solution $x(t)$ that satisfies the inequality $\|x(t)\| \leq h$.

In order to obtain the statement of the second result, some supplement assumption are needed. Suppose that $A(t, x), B_{i}(x)$ are $n \times n$ matrices given in the introduction that satisfy the conditions

$$
\begin{aligned}
& \left\|A(t, x)-A_{0}(x)\right\| \leq \delta, \quad\left\|B_{i}(x)-B_{i}^{0}\right\| \leq \delta_{1}, \\
& \max \left(\int_{0}^{T}\|F(t, x(t))\| d t, \sum_{i=1}^{p}\left\|c_{i}(x(t))\right\|\right) \leq N,
\end{aligned}
$$

where $t \in R,\|x(t)\| \leq h ; \delta, \delta_{1}$ are the positive constants given in (2.1) and $N$ shown in (2.4).

Theorem 2. Suppose that the equation system (1.4), (1.5) does not have nontrivial $T$ periodic solutions. Moreover, the differential equation system with impulsive effects (1.1), (1.2) satisfies conditions $(2.2),(2.5)$. Then the differential equation system with impulsive effects (1.1), (1.2) has at least one T-periodic solution.

\section{Proof of Theorems}

The proof of Theorem 1

i) The equation system (1.6), (1.7) can be rewritten according to the compare system (1.4), (1.5) as follows

$$
\begin{aligned}
& \frac{d X(t)}{d t}=A_{0}(t) X(t)+\left[A(t)-A_{0}(t)\right] X(t), \quad \forall t \neq \tau_{i}, \\
& \left.\Delta X\right|_{t=\tau_{i}}=B_{i}^{0} X\left(\tau_{i}\right)+\left[B_{i}-B_{i}^{0}\right] X\left(\tau_{i}\right) .
\end{aligned}
$$


Consequently, the matriciant of this system is of the form

$$
\begin{aligned}
X(t)=X_{0}(t) & +X_{0}(t) \int_{0}^{t} X_{0}^{-1}(s)\left[A(s)-A_{0}(s)\right] X(s) d s+ \\
& +\sum_{0<\tau_{i}<t} X_{0}(t) X_{0}^{-1}\left(\tau_{i}+0\right)\left[B_{i}-B_{i}^{0}\right] X\left(\tau_{i}\right) .
\end{aligned}
$$

After some uncomplicated transformations and by applying the extended Gronwell inequality, we obtain

$$
\left\|X(t)-X_{0}(t)\right\|_{0} \leq M L^{2} K^{3}\left(T \delta+p \delta_{1}\right)\left(1+L M K^{2} \delta_{1}\right)^{p} \exp \left(M L K^{2} T \delta\right) .
$$

From (1.14), (2.3) we have

$$
\left\|\left(H_{0}-H\right) x_{0}\right\|=\left\|V\left\{X_{0}(t)-X(t)\right\} x_{0}\right\| \leq 2\left\|X_{0}-X\right\|_{0}\left\|x_{0}\right\| \leq \frac{\rho}{\left\|H_{0}^{-1}\right\|}\left\|x_{0}\right\| .
$$

As consequence of the inequalities (2.1) and (2.3), it follows

$$
\rho\left\|x_{0}\right\| \geq\left\|H_{0}^{-1}\right\|\left\|\left(H_{0}-H\right) x_{0}\right\| \geq\left\|x_{0}\right\|-\left\|H_{0}^{-1}\right\|\left\|H x_{0}\right\| \geq\left\|x_{0}\right\|-\frac{\left\|H x_{0}\right\|}{\rho},
$$

for all $x \in R^{n}$. Hence

$$
\left\|H x_{0}\right\| \geq \rho(1-\rho)\left\|x_{0}\right\|, \quad \text { for all } x_{0} \in R^{n} .
$$

From here, it infers the existence of the inverse matrix $H^{-1}$, and the inequality (2.3) holds. form

ii) Every solution of the system of equations (1.6), (1.7) can be written in the following

$$
x(t)=-H^{-1}[V(p(t))]+\int_{0}^{t} A(s) x(s) d s+\int_{0}^{t} f(s) d s+\sum_{0<t_{1}<t} B_{i} x\left(\tau_{i}\right),
$$

where

$$
p(t)=X(t) \int_{0}^{t} X^{-1}(s) f(s) d s+\sum_{0<\tau_{i}<t} X(t) X^{-1}\left(\tau_{i}+0\right) c_{i} .
$$

It is estimated $\|X(t)\|$ and $\left\|X^{-1}(t)\right\|$. We have

$$
X(t)=E+\int_{0}^{t} A(s) X(s) d s+\sum_{0<\tau_{i}<t} B_{i} X\left(\tau_{i}\right) .
$$

After some uncomplicated calculations, we obtain

$$
\|X(t)\| \leq L_{1} K \exp (\delta T) .
$$


For the matrix $\left\|X^{-1}(t)\right\|$ we have

$$
X^{-1}(t)=E-\int_{0}^{t} X^{-1}(s) A(s) d s-\sum_{0<\tau_{i}<t} X^{-1}\left(\tau_{i}\right) B_{i}\left(E+B_{i}\right)^{-1} .
$$

Note that

$$
\begin{aligned}
& \left\|B_{i}\right\| \leq\left\|B_{i}^{0}\right\|+\left\|B_{i}-B_{i}^{0}\right\| \leq \beta_{i}+\delta_{1}, \\
& E+B_{i}=E+B_{i}^{0}+\left(B_{i}-B_{i}^{0}\right) .
\end{aligned}
$$

According to [4] given, the following estimation is obtained

$$
\left\|\left(E+B_{i}\right)^{-1}\right\| \leq\left\|\left(E+B_{i}^{0}\right)^{-1}\right\|+\frac{\left\|\left(E+B_{i}^{0}\right)^{-1}\right\|^{2}\left\|B_{i}-B_{i}^{0}\right\|}{1-\left\|\left(E+B_{i}^{0}\right)^{-1}\right\|\left\|B_{i}-B_{i}^{0}\right\|} \leq \ell_{i}+\frac{\ell_{i}^{2} \delta_{1}}{1-\ell_{i} \delta_{1}} .
$$

By applying the extended Gronwell inequality, the matrix $\left\|X^{-1}(t)\right\|$ is satisfying the inequality

$$
\left\|X^{-1}(t)\right\| \leq \prod_{i=1}^{p}\left(1+\frac{\ell_{i}\left(\beta_{i}+\delta_{1}\right)}{1-\ell_{i} \delta_{1}}\right) K \exp (T \delta)=M_{1} K \exp (T \delta) .
$$

We find the estimation for $\|p\|$. We have

$$
\begin{gathered}
\|p\| \leq\|X(t)\| \int_{0}^{T}\left\|X^{-1}(s)\right\|\|f(s)\| d s+\sum_{i=1}^{p}\|X(t)\|\left\|X^{-1}\left(\tau_{i}+0\right)\right\|\left\|c_{i}\right\|, \\
\|p\| \leq L_{1} M_{1} K^{2} \exp (T \delta) \int_{0}^{T}\|f(s)\| d s+L_{1} M_{1} K^{2} \exp (T \delta) \sum_{i=1}^{p}\left\|c_{i}\right\| \leq 2 L_{1} M_{1} K^{2} \exp (T \delta) N .
\end{gathered}
$$

From (3.3) we can write

$$
\begin{aligned}
\|x(t)\| & =\left\|H^{-1}\right\| \|\left[V(p(t)]\left\|+\int_{0}^{T}\right\| A(s)\|\| x(s)\left\|d s+\int_{0}^{T}\right\| f(s)\left\|d s+\sum_{i=1}^{p}\right\| B_{i}\|\| x\left(\tau_{i}\right) \|\right. \\
& \leq \frac{2 L_{1} M_{1} K^{2} \exp (T \delta) N}{\rho(1-\rho)}+N+\int_{0}^{T}\left(\left\|A_{0}(s)\right\|+\delta\right)\|x(s)\| d s+\sum_{i=1}^{p}\left(\left\|B_{i}^{0}\right\|+\delta_{1}\right) x\left(\tau_{i}\right) .
\end{aligned}
$$

Applying the extended Gronwell inequality, we obtain

$$
\begin{aligned}
\|x(t)\| & \leq \frac{2 L_{1} M_{1} K^{2} \exp (T \delta)+\rho(1-\rho)}{\rho(1-\rho)} N \prod_{i=1}^{p}\left(1+\beta_{i}+\delta_{1}\right) \times K \exp (T \delta) \\
& =\frac{2 L_{1} M_{1} K^{2} \exp (T \delta)+\rho(1-\rho)}{\rho(1-\rho)} N L_{1} K \exp (T \delta) .
\end{aligned}
$$

Since (2.4), it follows that $\|x(t)\| \leq h$. 


\section{The Proof of the Theorem 2}

Suppose that the functions $\varphi(t)$ are piecewise continuous, $T$-periodic with respect to $t$ $(t \in R)$ with the first kind discontinuities at $t=\tau_{i}, i=1,2, \ldots$ and belong to the sphere $S_{h}=\{\varphi(t):\|\varphi(t)\| \leq h\}$.

Let us consider the mapping $F \varphi$ defined by the form

$$
\begin{aligned}
& F \varphi=-H^{-1}\left[V\left(p_{A, F}(t)\right)\right]+p_{A, F}(t), \\
& p_{A, F}(t)=X_{A}(t) \int_{0}^{t} X_{A}^{-1}(s) F(s, \varphi(s)) d s+\sum_{0<\tau_{i}<t} X_{A}(t) X_{A}^{-1}\left(\tau_{i}+0\right) c_{i} .
\end{aligned}
$$

We show that $F$ maps the sphere $S_{h}$ into itself and continuous. Indeed, since $(2.2),(2.5)$, we get

$$
\begin{aligned}
& \left\|A(t, \varphi(t))-A_{0}(t)\right\| \leq \delta, \quad\left\|B_{i}\left(\varphi\left(\tau_{i}\right)\right) \varphi\left(\tau_{i}\right)-B_{i}^{0}\right\| \leq \delta_{1}, \\
& \max \left(\int_{0}^{T}\|F(t, \varphi(t))\| d t, \sum_{i=1}^{p}\left\|c_{i}\left(\varphi\left(\tau_{i}\right)\right)\right\|\right) \leq N .
\end{aligned}
$$

Thus, the matrices $A(t, \varphi(t)), B_{i}\left(\varphi\left(\tau_{i}\right)\right)$ and the vectors $F(t, \varphi(t)), c_{i}\left(\varphi\left(\tau_{i}\right)\right)$ satisfy all conditions of the Theorem 1, therefore, the vector-functions $F \varphi$ are also $T$-periodic in $t$, $t \in R$ piecewise continuous and belong to the sphere $S_{h}$ i.e. $F \varphi \in S_{h}$.

Now we will prove that the mapping $F \varphi$ is continuous i.e. it is shown that if the sequence $\varphi_{k}(t) \in S_{h}, k=1,2, \ldots$ is uniformly convergent to the function $\varphi_{\hat{0}}(t) \in S_{h}$, i.e. $\left\|\varphi_{k}(t)-\varphi_{\hat{0}}(t)\right\|_{0} \rightarrow 0$ then the sequence $F \varphi_{k}$ is also uniformly convergent to the function $F \varphi_{\hat{0}}$, i.e. $\left\|F \varphi_{k}(t)-F \varphi_{\hat{0}}(t)\right\|_{0} \rightarrow 0$. It is clearly that $A\left(\varphi_{k}(t), t\right), F\left(\varphi_{k}(t), t\right), B_{i}\left(\varphi_{k}(t)\right)$ respectively uniformly converge to $A\left(\varphi_{\hat{0}}(t), t\right), F\left(\varphi_{\hat{0}}(t), t\right), B_{i}\left(\varphi_{\hat{0}}(t)\right)$.

Denote that $X_{k}(t), X_{\hat{0}}(t)$ respectively are the matriciants of the system of equations

$$
\begin{aligned}
& \dot{x}=A\left(\varphi_{k}(t), t\right) x, \quad t \neq \tau_{i}, \\
& \left.\Delta x\right|_{t=\tau_{i}}=B_{i}\left(\varphi_{k}(t)\right) x
\end{aligned}
$$

and

$$
\begin{aligned}
& \dot{x}=A\left(\varphi_{\hat{0}}(t), t\right) x, \quad t \neq \tau_{i}, \\
& \left.\Delta x\right|_{t=\tau_{i}}=B_{i}\left(\varphi_{\hat{0}}(t)\right) x .
\end{aligned}
$$

Similarly to the estimate (3.1), the difference $\left\|X_{k}(t)-X_{\hat{0}}(t)\right\|_{0}$ satisfies the following estimation:

$$
\begin{aligned}
& \left\|X_{k}(t)-X_{\hat{0}}(t)\right\|_{0} \\
& \leq M_{\hat{0}} L_{\hat{0}}^{2} K_{\hat{0}}^{3}\left(T\left\|A\left(\varphi_{k}(t), t\right)-A\left(\varphi_{\hat{0}}(t), t\right)\right\|_{0}+p\left\|B_{i}\left(\varphi_{k}\left(\tau_{i}\right)\right)-B_{i}\left(\varphi_{\hat{0}}\left(\tau_{i}\right)\right)\right\|\right) \\
& \times\left(1+L_{\hat{0}} M_{\hat{0}} K_{\hat{0}}^{2}\left\|B_{i}\left(\varphi_{k}\left(\tau_{i}\right)\right)-B_{i}\left(\varphi_{\hat{0}}\left(\tau_{i}\right)\right)\right\|\right)^{p} \\
& \times \exp \left(M_{\hat{0}} L_{\hat{0}} K_{\hat{0}}^{2} T\left\|A\left(\varphi_{k}(t), t\right)-A\left(\varphi_{\hat{0}}(t), t\right)\right\|_{0}\right),
\end{aligned}
$$


where $L_{\hat{0}}, M_{\hat{0}}, K_{\hat{0}}$ are the constants similar to the respective constants introduced in (1.14). It follows sequence $X_{k}(t)$ uniformly converges to the limit matrix $X_{0}(t)$ as $k \rightarrow \infty$. Now, the symbols $H_{k}$ và $H_{\hat{0}}$ are introduced by the forms

$$
H_{k}=I-X_{k}(T), \quad H_{\hat{0}}=I-X_{\hat{0}}(T) .
$$

Hence

$$
\left\|H_{k}-H_{\hat{0}}\right\|=\left\|X_{k}(T)-X_{\hat{0}}(T)\right\| \leq\left\|X_{k}(t)-X_{\hat{0}}(t)\right\|_{0},
$$

therefore $\left\|H_{k}-H_{\hat{0}}\right\| \rightarrow 0$ as $k \rightarrow \infty$.

According to Theorem 1 , there are inverse operators $H_{k}^{-1}$ and $H_{0}^{-1}$ satisfying the condition

$$
\begin{aligned}
& \left\|H_{k}^{-1}\right\| \leq \frac{1}{\rho(1-\rho)}, \quad\left\|H_{0}^{-1}\right\| \leq \frac{1}{\rho(1-\rho)}, \quad \text { and so } \\
& \left\|H_{k}^{-1}-H_{\hat{0}}^{-1}\right\| \leq\left\|H_{k}^{-1}\right\|\left\|H_{k}-H_{\hat{0}}\right\|\left\|H_{\hat{0}}^{-1}\right\| \leq \frac{\left\|X_{k}-X_{\hat{0}}\right\|}{\rho^{2}(1-\rho)^{2}} .
\end{aligned}
$$

Thus $\left\|H_{k}^{-1}-H_{\hat{0}}^{-1}\right\|$ is approximate to żero for $k \rightarrow \infty$.

Note that $X_{k}^{-1}, X_{\hat{0}}^{-1}$ are respectively the matriciants of the systems of equations

$$
\begin{aligned}
& \frac{d X_{k}^{-1}}{d t}=X_{k}^{-1} A_{k}(t), \quad t \neq \tau_{i}, \\
& \left.\Delta X_{k}^{-1}\right|_{t=\tau_{i}}=X_{k}^{-1}(t) B_{i k}\left(E+B_{i k}\right)^{-1},
\end{aligned}
$$

where $A_{k}(t)=A\left(\varphi_{k}(t), t\right), B_{i k}=B_{i}\left(\varphi_{k}\left(\tau_{i}\right)\right)$, and

$$
\begin{aligned}
& \frac{d X_{\hat{0}}^{-1}}{d t}=X_{\hat{0}}^{-1} A_{\hat{0}}(t), \quad t \neq \tau_{i}, \\
& \left.\Delta X_{\hat{0}}^{-1}\right|_{t=\tau_{i}}=X_{\hat{0}}^{-1}(t) B_{i \hat{0}}\left(E+B_{i \hat{0}}\right)^{-1},
\end{aligned}
$$

with $A_{\hat{0}}(t)=A\left(\varphi_{\hat{0}}(t), t\right), B_{i \hat{0}}=B_{i}\left(\varphi_{\hat{0}}\left(\tau_{i}\right)\right)$.

Consequently we can write

$$
\begin{aligned}
X_{k}^{-1}(t)= & X_{\hat{0}}^{-1}(t)-\left\{\int_{0}^{t} X_{k}^{-1}(s)\left[A_{\hat{0}}(s)-A_{k}(s)\right] X_{\hat{0}}^{-1}(s) d s\right\} X_{\hat{0}}^{-1}(t) \\
& -\sum_{0<\tau_{i}<t} X_{k}^{-1}\left(\tau_{i}\right)\left[\left(B_{i k}-B_{i \hat{0}}\right)\left(E+B_{i \hat{0}}\right)^{-1}\right. \\
& \left.+B_{i k}\left(\left(E+B_{i k}\right)^{-1}-\left(E+B_{i \hat{0}}\right)^{-1}\right)\right] X_{\hat{0}}^{-1}\left(\tau_{i}+0\right) X_{\hat{0}}^{-1}(t) .
\end{aligned}
$$

From here it follows that $X_{k}^{-1}(t)$ uniformly converges to $X_{\hat{0}}^{-1}(t)$ for $k \rightarrow \infty$. 
From all argument given above, it deduces that the sequence $F \varphi_{k}$ :

$$
\begin{aligned}
& F \varphi_{k}=-H_{k}^{-1}\left[V\left(p_{A_{k}, F_{k}}(t)\right)\right]+p_{A_{k}, F_{k}}(t) \\
& p_{A_{k}, F_{k}}(t)=X_{k}(t) \int_{0}^{t} X_{k}^{-1}(s) F\left(s, \varphi_{k}(s)\right) d s+\sum_{0<\tau_{i}<t} X_{k}(t) X_{k}^{-1}\left(\tau_{i}+0\right) c_{k}^{i},
\end{aligned}
$$

will uniformly converge to $F \varphi_{\hat{0}}$ :

$$
\begin{aligned}
& F \varphi_{\hat{0}}=-H_{\hat{0}}^{-1}\left[V\left(p_{A_{\hat{0}}, F_{\hat{0}}}(t)\right)\right]+p_{A_{\hat{0}}, F_{\hat{0}}}(t), \\
& p_{A_{\hat{0}}, F_{\hat{0}}}(t)=X_{\hat{0}}(t) \int_{0}^{t} X_{\hat{0}}^{-1}(s) F\left(s, \varphi_{\hat{0}}(s)\right) d s+\sum_{0<\tau_{i}<t} X_{\hat{0}}(t) X_{\hat{0}}^{-1}\left(\tau_{i}+0\right) c_{\hat{0}}^{i} .
\end{aligned}
$$

Thus, the mapping $F \varphi$ is continuous. To end the proof we will show that the set $F S_{h}$ in the space of $T$-periodic, piecewise continuous with the first kind discontinuities at $t=\tau_{i}$ functions given above has a compact closure, i.e. any sequence $\varphi_{1}, \varphi_{2}, \ldots$ of the set $S_{h}$ has a convergence subsequence. Indeed, suppose that $\left\{\varphi_{k}\right\}$ is an arbitrary sequence of $S_{h}$. This sequence is uniformly bounded and equicontinuous on the interval $\left[0, \tau_{1}\right]$, and so, by Arzela theorem, there is a subsequence $\left\{\varphi_{k}^{(1)}\right\}$ uniformly convergent on $\left[0, \tau_{1}\right]$. Consider this subsequence on $\left(\tau_{1}, \tau_{2}\right]$ we also can select a subsequence $\left\{\varphi_{k}^{(2)}\right\}$ uniformly convergent on $\left(\tau_{1}, \tau_{2}\right]$. Continuing this process for the intervals $\left(\tau_{2}, \tau_{3}\right],\left(\tau_{3}, \tau_{4}\right], \ldots\left(\tau_{p}, T\right]$ of $[0, T]$ we get a subsequence $\left\{\varphi_{k}^{(p+1)}\right\}$ uniformly convergent on the whole interval $[0, T]$. This means that, $F S_{h}$ has a compact closure in considering banach space, so by theorem on existence of a fixed point of an operator on a complete normed space, we conclude that $F \varphi$ has a fixed point in $S_{h}$, i.e. the system (1), (2) has at least one $T$-periodic solution. Therefore, the theorem is completely proved.

Acknowledgment. Support from the National Fundamental Research Program in Natural Sciences is gratefully acknowledged.

\section{References}

1. Kartsatos A. G., Advanced Differential Equations, Mariner, Tampa, Florida, 1980.

2. Saito S. and Yamamoto M., Periodic solutions for the periodic quasilinear ordinary differential equations system, Math. Japanica 34 (1) (1989) 111-121.

3. Samoilenko A. M. and Peretyuk, Impulsive Differential Equations, World Scientific, 1995.

4. Roger A. Horn and Charles R. Johnson, Matrix Analysis, Cambridge University Press, 1986. 


\section{VÊ SỰ TỒN TAI NGHIÊMM TUẦN HOẢN CƯA MỘT SỐ PHƯONG TRİNH VI PHÂN TỰ TUYẾN TÍNH VỚI TÁC DỤNG XUNG}

Trong công trình này, ta khảo sát bài toán về sự tồn tại nghiệm tuần hoàn của một lớp các hệ phương trình vi phân tựa tuyến tính chịu tác dụng xung có dạng

$$
\begin{aligned}
& \dot{x}=A(x, t) x+F(t, x), \quad t \neq \tau_{i}, \\
& \left.\Delta x\right|_{t=\tau_{i}}=B_{i}(x) x+c_{i}(x), \\
& B_{i+p}(x)=B_{i}(x), \quad c_{i+p}=c_{i}(x), \quad p \text {-nguyên, } \quad i=1,2, \ldots,
\end{aligned}
$$

Cùng với (1.1), (1.2), (1.3) ta khåo sát hệ tuyến tính

$$
\begin{aligned}
& \dot{x}=A_{0}(t) x, \quad t \neq \tau_{i}, \\
& \left.\Delta x\right|_{t=\tau_{i}}=B_{i}^{0} x, \quad B_{i+p}^{0}=B_{i}^{0}, \quad i=1,2, \ldots
\end{aligned}
$$

Đã chứng minh sự tồn tại nghiệm tuần hoàn của bài toán $(1.1),(1.2),(1.3)$ nếu vế phải thỏa mãn những điều kiện định lượng xác định. 